\title{
Chaotic S-Box: Intertwining Logistic Map and Bacterial Foraging Optimization
}

\author{
Ye Tian ${ }^{1,2}$ and Zhimao $\mathrm{Lu}^{1,3}$ \\ ${ }^{1}$ College of Information and Communication Engineering, Harbin Engineering University, Harbin 150001, China \\ ${ }^{2}$ Key Laboratory of Photonic and Electronic Bandgap Materials, Ministry of Education, School of Physics and Electronic Engineering, \\ Harbin Normal University, Harbin 150025, China \\ ${ }^{3}$ Faculty of Electronic Information and Electrical Engineering, Dalian University of Technology, Dalian 116024, China
}

Correspondence should be addressed to Ye Tian; hsdtianye@126.com

Received 16 June 2017; Revised 24 October 2017; Accepted 26 October 2017; Published 15 November 2017

Academic Editor: Maria L. Gandarias

Copyright ( 2017 Ye Tian and Zhimao Lu. This is an open access article distributed under the Creative Commons Attribution License, which permits unrestricted use, distribution, and reproduction in any medium, provided the original work is properly cited.

As the unique nonlinear component of block ciphers, Substitution box (S-box) directly affects the safety of a cryptographic system. It is important and difficult to design strong S-box that simultaneously meets multiple cryptographic criteria such as bijection, nonlinearity, strict avalanche criterion (SAC), bit independence criterion (BIC), differential probability (DP), and linear probability (LP). Though many chaotic S-boxes have been proposed, the cryptographic performance of most of them needs to be further improved. A new chaotic S-box based on the intertwining logistic map and bacterial foraging optimization is designed in this paper. It firstly iterates the intertwining logistic map to construct many S-boxes and then applies a bacterial foraging optimization algorithm to find the optimal S-box. Moreover, bacterial foraging optimization algorithm considers the nonlinearity and differential uniformity as the fitness functions in the optimization process. We experiment that the proposed S-box can effectively resist multiple types of cryptanalysis attacks.

\section{Introduction}

The dynamic developments in the multimedia industry and the Internet lead to a considerable amount of worry regarding the security of information transmitted over open or stored channels [1-3]. How to protect information from being unauthorized handled is becoming extremely crucial. Modern cryptography technique, in which block cipher algorithm is an important research direction, is an effective way to guarantee the safety of information, since then many researchers have developed a lot of block cipher algorithms. In a block cipher algorithm, Substitution box (S-box) is the only one nonlinear component [4], providing the block cipher system with necessary confusing and scrambling effect against attacks. Moreover, its cryptography security features directly determine the safety of the entire cipher performance [1]. Mathematically, an $n \times n$ size of S-box is a nonlinear mapping $S:\{0,1\}^{n} \rightarrow\{0,1\}^{n}$, where $\{0,1\}^{n}$ represents the vector spaces of $n$ elements from GF(2), and we set $n=8$ in this paper.
Many papers on S-boxes have been published by scholars around the world over the past decades. In [5], Hussain and Gondal presented a design approach for $n \times n$ S-boxes, which was an exhaustive search method; nevertheless, the performance of this procedure would become rather difficult with the increase of $n$. Liu et al. [4] utilized near-bent Boolean functions of five variables to generate $5 \times 5$ S-boxes to resist the differential attack; however, their algorithm was useful only to create an S-box of odd input bit number. Therefore, most of these approaches were inefficient and were unable to construct S-boxes that could meet multiple assessment requirements simultaneously.

Chaotic systems that satisfy the major requirements of cryptography properties such as diffusion and confusion are differentiated on the basis of their reactiveness to ergodicity, pseudorandomness, unpredictability, control parameters, and initial conditions; this makes chaotic systems particularly catch heaps of heed for cryptology [1-5]. Due to this matter, chaotic S-boxes have proved to be superior for encrypting a message. 
For example, literature [6] proposed a four-step method of generating chaotic S-box based on discrete logistic map. It turned out that very simple chaotic maps and discretization procedure generated secure S-boxes. Literature [7] improved the work in [6] using bit extraction and Baker map. Furthermore, literature [8] proposed an S-box design approach based on iteration discrete chaotic that had high immunity to the differential cryptanalysis. Using three-dimensional chaotic Baker map, literature [9] constructed an S-box that approximately fulfilled all the criteria for a cryptographically strong S-box.

However, a simple chaos system also has many defects; for example, the implementation of the chaos on a computer is affected by the limited precision; the time series outputted by the simple chaotic system generally cannot reach the theoretically complete random, resulting in the problem that the pseudorandom sequence appears periodicity [10].

To cope with these problems, many complex chaotic maps based S-boxes have been presented in recent years. For example, literature [11] indicated the pseudorandomness and complexity of binary sequences produced by the Lorenz system and Chebyshev map. Literature [12] designed a new pseudorandom number generator by mixing the couple map lattice technology and the chaos iteration technology. Literature [13] proposed a secure pseudorandom number generator three-mixer. Khan et al. presented a complex chaotic S-box construction method that could provide better security in terms of resistance against various attacks by deploying the 2D Henon chaotic map and skew tent map [14]. Ahmad et al. proposed a method for synthesizing cryptographically efficient chaotic S-box, which integrated four 1D chaotic systems, namely, logistic maps and cubic maps, to modulate the normal system trajectories of the other [15]. Peng et al. designed a novel approach for dynamically generating Sboxes using a spatiotemporal chaotic system, which mapped the key to system parameters and generated the hyperchaotic sequences to construct S-boxes [16].

By increasing the complexity of the chaotic system, these complex algorithms obtained S-boxes with the higher security level to some extent. Nevertheless, the performance gap between many of these chaotic S-boxes and classic ones still exists; for example, few chaos-based S-boxes can achieve the high performance like the one used in advanced encryption standard (AES) [17].

Compared with other intelligent optimization algorithms, bacterial foraging optimization algorithm (BFO) has a group of intelligence and can carry out parallel search. Besides, it may be easy to jump out of the local minimal solution; thus, it can find solutions of higher quality. Due to these advantages, it has been widely used in some research fields. For example, Abd-Elazim and Ali proposed an optimization algorithm BFOA for controlling the damping of the power system's electromechanical oscillations [18]. On the basis of literature [18], Ali and Abd-Elazim proposed a BFOA based Load Frequency Control (LFC) for the suppression of oscillations in power system [19]. In addition, Abd-Elazim and Ali developed an optimization algorithm BSO, which synergistically coupled the BFOA with the particle swarm optimization algorithm for the optimal design of the TCSC damping controller. Specifically, they transformed the controller design problem into an optimization problem, and the BSO was developed to find the optimal controller parameters [20]. Sur and Shukla also presented a discrete adaptive BFO algorithm, which could be applied to discrete search domains and various multidimensional problems [21]. Furthermore, to optimize a power network problem, Tripathy and Mishra proposed an improved $\mathrm{BFO}$ algorithm. In this work, the power network problem was formulated as a multiobjective multivariable problem, and the improved BFO was applied to solve this problem [22].

In this paper, a new scheme for designing an S-box is presented. Unlike other chaos-based algorithms that generate strong S-boxes by using the random distribution property of chaotic maps, we divide the process of designing an S-box into two steps. Firstly, we generate many S-boxes by iterating the chaotic map. Secondly, we apply a genetic algorithm, the evaluation function of which adopts the nonlinearity and differential uniformity to improve the performance of the generated S-box. We show via simulation that our scheme can generate stronger S-box. Abbreviations section shows some abbreviations of technical terms involved in this paper.

\section{Preliminary Work}

2.1. Intertwining Logistic Map. In this section, we will introduce a chaotic map, the intertwining logistic map [23], which is defined as follows:

$$
\begin{aligned}
& x_{n+1}=\left[\mu \times k_{1} \times y_{n} \times\left(1-x_{n}\right)+z_{n}\right] \bmod 1 \\
& y_{n+1}=\left[\frac{\mu \times k_{2} \times y_{n}+z_{n} \times 1}{\left(1+\left(x_{n+1}\right)^{2}\right)}\right] \bmod 1 \\
& z_{n+1}=\left[\mu \times\left(x_{n+1}+y_{n+1}+k_{3}\right) \times \sin \left(z_{n}\right)\right] \bmod 1,
\end{aligned}
$$

where $\mu, k_{i}$ are the system parameters with the ranges $0<$ $\mu<3.999,\left|k_{1}\right|>33.5,\left|k_{2}\right|>37.9,\left|k_{3}\right|>35.7$. Figures 1(a), $1(\mathrm{~b})$, and 1 (c) show the chaotic bifurcation diagrams of the intertwining Logistic map when $k_{1}=39.7, k_{2}=40.2, k_{3}=$ 38.5. Figure $1(d)$ shows the chaotic bifurcation diagram of the logistic map. Figure 2(a) depicts the chaotic attractor diagram of the intertwining Logistic map when $k_{1}=39.7, k_{2}=40.2$, $k_{3}=38.5$, and $\mu=1.5$. Figure 2(b) depicts the chaotic attractor diagram of the logistic map. In the intertwining logistic map system, the sequence distribution becomes more uniform, and, more importantly, empty windows are eliminated. Remarkably, comparing with a simple logistic map, the action of a intertwining logistic chaotic map is more complex, and the sequence distribution of it is more uniform.

2.2. Bacterial Foraging Optimization Algorithm. In 2002, Passino proposed a bacterial foraging optimization algorithm that imitated groups competition coordination mechanism of Escherichia coli (E. coli) in the process of searching for food in the human gut. In looking for the region of food sources, E. coli might determine whether they enter the region through a priori knowledge. Once entering foraging area, if a certain amount of food was consumed or foraging 


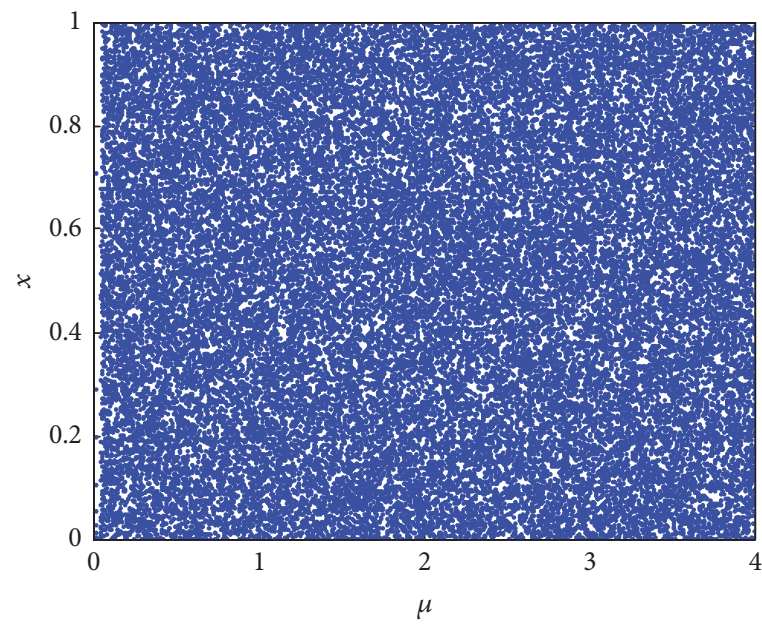

(a) Bifurcation diagram of a intertwining logistic map $x$

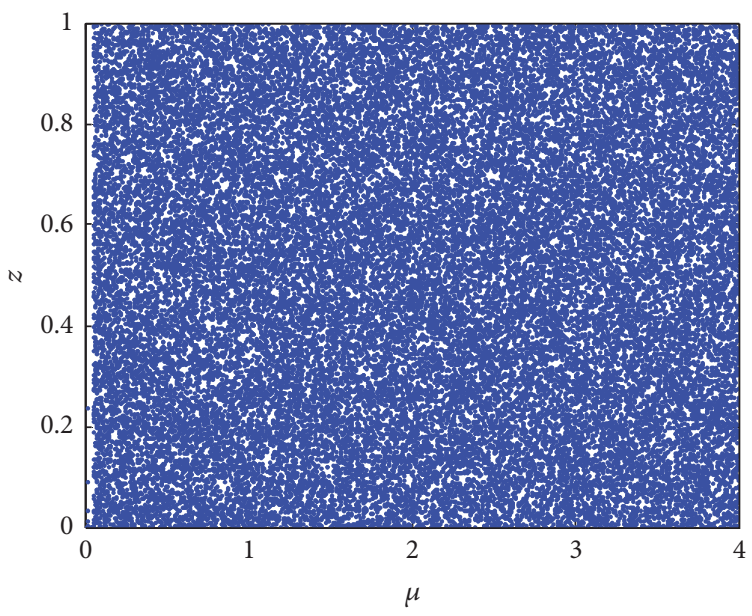

(c) Bifurcation diagram of a intertwining logistic map $z$

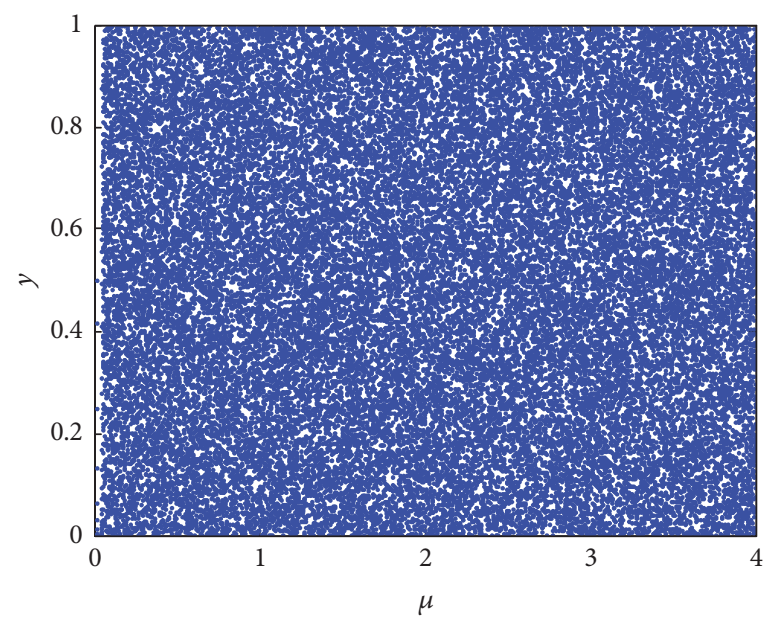

(b) Bifurcation diagram of a intertwining logistic map $y$

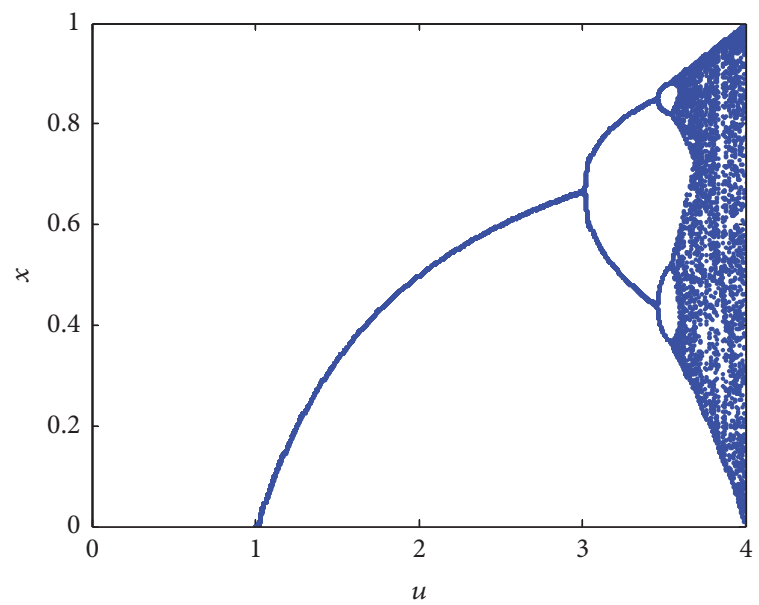

(d) Bifurcation diagram of a logistic map

FIGURE 1: Bifurcation diagrams comparisons of the logistic map and intertwining logistic map.

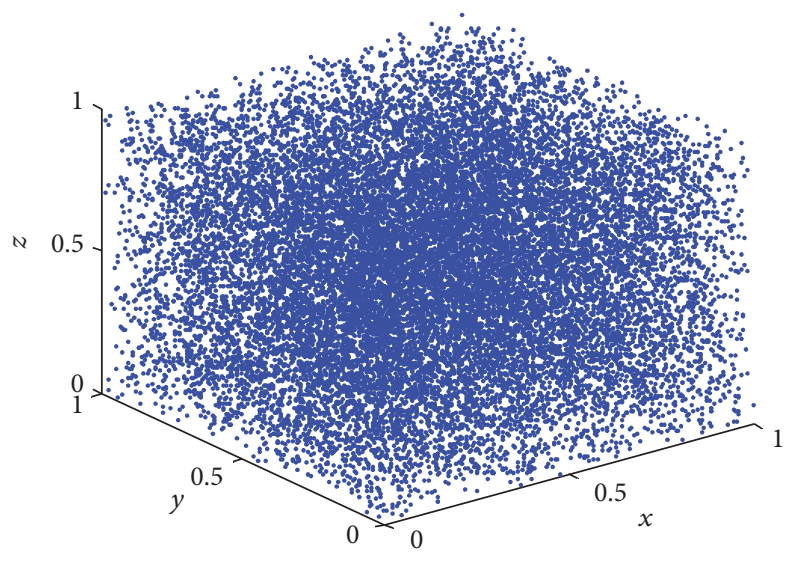

(a) Attractor of a intertwining logistic map

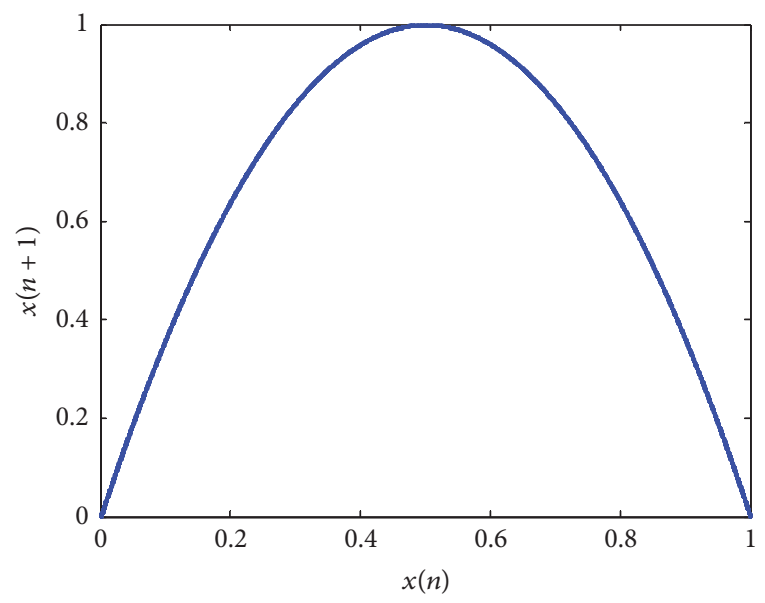

(b) Attractor of a logistic map

FIGURE 2: Attractor comparisons of the logistic map and intertwining logistic map. 
region environment deteriorated, resulting in inhospitable conditions, a bacteria might die or move to another suitable region. Relying on its own unique behaviors, chemotaxis, reproduction, and elimination-dispersal, bacterial foraging algorithm proceeded to update bacteria individual position and searched the optimal locations of bacteria groups, so as to realize the evolution of the population. The main steps of bacterial foraging optimization algorithm can be described as follows [24].

Step 1 (parameters initialization). Ned is the number of elimination-dispersal events; Nre is the number of reproduction steps. Nc is the number chemotaxis steps. Ped is the basic probability of elimination-dispersal. SS is the bacteria scale. Ns is the maximum number of chemotactic steps.

Step 2. Initialize the bacteria locations; calculate the initialization fitness values of bacteria.

Step 3. Perform the elimination-dispersal loop $l=1:$ Ned, reproduction loop $k=1$ : Nre, and chemotaxis loop $j=1$ : Nc.

Step 4 (perform bacteria chemotaxis loop). Use $X_{i}(j, k, l)$ to express the space location vector of bacteria, where $j$ represents the $j$ th generation of chemotaxis loop, $k$ represents the $k$ th generation of reproduction loop, and $l$ represents the $l$ th generation of elimination-dispersal loop.

(1) Tumble. Update bacteria locations by

$$
\begin{aligned}
X_{i}(j+1, k, l)=X_{i}(j, k, l) & +C(i) \times \phi(i, j) \\
\phi(i, j) & =\frac{\Delta(i, j)}{\sqrt{\Delta^{T}(i, j) \Delta(i, j)}},
\end{aligned}
$$

where $C(i)$ is the chemotaxis step length of bacterium $i . \phi(i, j)$ is an normalized random direction vector of bacterium $i$ when tumbling in the $j$ th loop. $\Delta(i, j)$ is arbitrarily generated random direction vector with each element being a random number on $[-1,1]$.

(2) Move. If the fitness value of tumbling improves, it will not move according to the direction of tumbling until the fitness value no longer improves or reaches the maximum moves steps Ns.

Step 5 (reproduction loop). After completing the chemotaxis loop, accumulate the fitness values of each bacterium during its life cycle to get an energy value. Order bacteria by the energy values; eliminate half bacteria which are poor to obtain energy. Reproduce half bacteria that have strong ability of energy harvesting.

Step 6 (elimination-dispersal loop). After completing the reproduction operator, generate a random probability, and compare it with the fixed elimination-dispersal probability Ped. If the generated probability is lower than Ped, proceed with the bacteria elimination-dispersal, and random initialize in the definition domain of the solution space.
Step 7. Determine the loop end condition; if it meets the condition, end and output the results; otherwise, continue.

\section{Scheme of Generating of an S-Box}

3.1. Improved Bacteria Locations Update Equation. Bacteria foraging optimization algorithm was designed to solve the problem of continuous optimization. The map among different S-boxes, however, belongs to a discrete problem; thus we will firstly operate the BFO discretization. To realize it, we introduce a concept of commutator [42] in this paper.

The commutator denoted by $Z=\operatorname{Swap}(x, y), x, y \in$ $\{1,2,3, \ldots, N\}$, where $N$ represents the number of elements of a food source location, represents the exchange between $x$ and $y$ in a food location. Based on one solution, exchange any two solutions several times to obtain another solution. The difference between these two solutions can be denoted by a swap sequence $\left\{Z_{1}, Z_{2}, \ldots, Z_{m}\right\}$, where $m$ is the number of commutators.

Next, we should define an operator operation symbol: assume two different solutions $X$ and $Y$; define $X-Y=$ $\left\{Z_{1}, Z_{2}, \ldots, Z_{m}\right\}$; that is, the difference of $X$ and $Y$ determines the exchange sequence; the physical meaning of the operation is that the solution $Y$ will be the same as $X$ after operating $m$ different commutators. The definition of the operation $X+\left\{Z_{1}, Z_{2}, \ldots, Z_{m}\right\}$ can be described as follows: exchange the solution $X,\left\{Z_{1}, Z_{2}, \ldots, Z_{m}\right\}$ times, and connect these two commutators sequences one after another, respectively. For example, $X_{i}(t)=[1,2,3,4,5,6,7,8]$, $X_{k}(t)=[8,6,5,1,4,7,2,3], V(t)=X_{k}(t)-X_{i}(t)=$ $\{(1,8)(2,6)(3,5)(4,1)(3,4)(2,7)\}$. Change $(2)$ by $X_{i}(t+$ $1)=X_{i}(t)+C \times \operatorname{rand}()\left(X_{i}(t)-X_{k}(t)\right)$, where $\operatorname{rand}()$ is a random number on $[0,1], C$ is the speed adjustment parameter, and $\alpha$ is a constant ranging of $[0,1]$. Furthermore, if $C \times \operatorname{rand}()$ is lower than $\alpha$, retain the commutator.

3.2. Fitness Function. In the BFO, fitness function described the quality of a feasible solution which refers to the richness of the food sources and directly relates to the optimization results. When utilizing chaos optimal search method to construct an S-box, since an S-box has more than one Boolean function characteristic, we should construct a weighted equation to weigh the different cryptography characteristics of the S-box.

As the linear and differential analyses are two effective ways of attack analysis, we will consider the nonlinearity and differential uniformity of an S-box when designing a fitness function that can take the following form:

$$
f(s)=a_{s} f_{s}\left(N_{s}\right)+a_{d} f_{d}\left(\delta_{s}\right)
$$

where the nonlinearity function is $f_{s}\left(N_{s}\right)=N_{s}$; the differential uniformity function is $f_{d}\left(\delta_{s}\right)=\delta_{s}$.

Fitness function should satisfy the monodromy and continuity. According to the experimental results, we order $\left(N_{s}, \delta_{s}\right)$ by the performance of the S-box, that is, $(108,6)$, $(108,8),(106,6),(106,8),(108,10)$, and $(106,10)$ in this paper. Moreover, we assign $a_{s}$ and $a_{d}$ and obtain the fitness function as follows [43]: 


$$
f(s)= \begin{cases}N_{s}-\delta_{s} & N_{s}<90, \delta_{s}>18 \\ N_{s}-2 \delta_{s} & N_{s}<90, \delta_{s} \leq 18 \\ \frac{3}{2} N_{s}-\delta_{s} & N_{s} \geq 90, \delta_{s}>18 \\ \frac{3}{2} N_{s}-2 \delta_{s} & N_{s} \geq 90, \delta_{s} \leq 18\end{cases}
$$

3.3. The Main Steps of Generating an S-Box. According to the above description, we can summarize the detailed steps of generating an S-box as follows.

Step 1. Call the parameters initialization of the BFO, set a speed adjustment parameter $C$ and constant $\alpha$, substitute the initial values, $x_{0}, y_{0}, z_{0}$, into (1), iterate $N_{0}$ times to obtain $x_{1}$, $y_{1}, z_{1}$, and define an integer array $\mathbf{S}$ of length 256.

Step 2. Take $x_{1}, y_{1}, z_{1}$, as the initial values, record from $\left(N_{0}+1\right)$ th value, and mark the following obtained real-value sequences, $x_{i}, y_{i}, z_{i}(i=1,2, \ldots)$.

Step 3. Convert these continuous real-value sequences $x_{i}$, $y_{i}, z_{i}$, to the corresponding integer sequences by (5), with $\left\{s x_{i}, s y_{i}, s z_{i}\right\} \in[0,255]$, and sort them by (6).

$$
\begin{aligned}
& s x_{i}=\left\lfloor x_{i} \times 256\right\rfloor \\
& s y_{i}=\left\lfloor y_{i} \times 256\right\rfloor \\
& s z_{i}=\left\lfloor z_{i} \times 256\right\rfloor,
\end{aligned}
$$

where $\lfloor x\rfloor$ returns the maximum integer less than or equal to $x$.

$$
\begin{aligned}
& Q=\left\{\begin{array}{lll}
s x_{i} & s y_{i} & s z_{i}
\end{array}\right\} \\
& R=\left\{\begin{array}{lll}
s y_{i} & s z_{i} & s x_{i}
\end{array}\right\} \\
& T=\left\{\begin{array}{lll}
s z_{i} & s x_{i} & s y_{i}
\end{array}\right\} .
\end{aligned}
$$

Step 4. Iterate $M_{0}$ and record starting from $\left(M_{0}+1\right)$ th value; if $Q_{i}$ has appeared in array $S_{i}$, discard it; otherwise, save it in $S_{i}$. And the $S_{i}$ box is obtained when the array is filled. So are $R_{i}$ and $T_{i}$. Iterate $M_{0} k$ and record from the $\left(M_{0} k+1\right)$ th value; if $Q_{i}$, has appeared in the array $S_{i}$, discard it; otherwise, save it in $S_{i}$ until the array is filled. So are $R_{i}$ and $T_{i}$. Similarly, iterate $M_{0} 2 k$, record from $\left(M_{0} 2 k+1\right)$ th value, and obtain $Q_{i}, R_{i}$, and $T_{i}$.

Step 5. Arrange the integer sequence $S_{i}$ in a $2^{n / 2} \times 2^{n / 2}$ table, and construct $3 k$ S-boxes.

Step 6. Calculate the fitness value of each S-box by (3).

Step 7. Call Steps 3-7 of BFO, except that we use (7) to update the bacteria locations in the tumbling stage.

$$
X_{i}(t+1)=X_{i}(t)+C \times \operatorname{rand}() *\left(X_{i}(t)-X_{k}(t)\right) .
$$

\section{S-Box Evaluation Criteria}

To obtain the S-box with desired cryptography properties, many evaluating criteria, such as bijectivity, Nonlinearity,
SAC, BIC, DP, and LP, have been designed. We will also use these criteria to test the performance of the proposed S-box in this paper.

4.1. Bijectivity. Adams and Tavares pointed out that if the linear sum of the Boolean function $f_{i}$ of each component of the designed $n \times n$ S-box was $2^{n-1}, f$ was then a bijection [44]. Specifically, the expression is as follows:

$$
\mathrm{wt}\left(\sum_{i=1}^{n} a_{i} f_{i}\right)=2^{n-1} .
$$

Here, $a_{i} \in\{0,1\},\left(a_{1}, a_{2}, \ldots, a_{n}\right) \neq(0,0, \ldots, 0)$, wt( ) denotes the Hamming weight.

In fact, a reversible S-box is usually required; especially in a replacement network, the $\mathrm{S}$-box must be bijective.

\subsection{Nonlinearity}

Definition 1. Let $f(x): F_{2}^{n} \rightarrow F_{2}$ be an $n$ Boolean function; the nonlinearity of $f(x)$ can take the following form:

$$
H_{f}=\min _{l \in L_{n}} d_{H}(f, l),
$$

where $L_{n}$ is a set of the whole linear and affine functions and $d_{H}(f, l)$ denotes the Hamming distance between $f$ and $l$.

The nonlinearity denoted by the Walsh spectrum can take the following form:

$$
N_{f}=2^{-n}\left(1-\max _{\omega \in \mathrm{GF}\left(2^{n}\right)}\left|S_{\langle f\rangle}(\omega)\right|\right) .
$$

The cyclic spectrum of the function $f(x)$ is

$$
S_{\langle f\rangle}(\omega)=2^{-n} \sum_{x \in \mathrm{GF}\left(2^{n}\right)}(-1)^{f(x) \oplus x \cdot \omega}
$$

where $\omega \in \operatorname{GF}\left(2^{n}\right)$ and $x \cdot \omega$ denotes the dot product of $x$ and $w$.

The larger the nonlinearity $N_{f}$ of the function $f$, the stronger the ability of its resistance to the linear attacks, and vice versa.

4.3. Strict Avalanche Criterion. Strict avalanche criterion describes a fact that when one bit in the input of Boolean function changes, the changing probability of every bit in its output is $1 / 2$. In practical application, a correlation matrix, the construction method of which refers to literature [45], is always constructed to test the SAC property of the Boolean function.

4.4. Bit Independent Criterion. Given that a Boolean function $f_{j}, f_{k}(j \neq k)$ is a two-bit output of an S-box, if $f_{j} \oplus f_{k}$ is highly nonlinear and meets the SAC, the correlation coefficient of each output bit pair may be close to 0 when one input bit is inversed. Thus, we can check the BIC of the S-box by verifying whether $f_{j} \oplus f_{k}(j \neq k)$ of any two output bits of the S-box meets the nonlinearity and SAC [45]. 
TABLE 1: The generated S-box.

\begin{tabular}{|c|c|c|c|c|c|c|c|c|c|c|c|c|c|c|c|c|}
\hline Number & $(1)$ & $(2)$ & $(3)$ & $(4)$ & $(5)$ & $(6)$ & $(7)$ & $(8)$ & (9) & $(10)$ & $(11)$ & (12) & (13) & (14) & $(15)$ & (16) \\
\hline (1) & 88 & 1 & 229 & 243 & 165 & 197 & 249 & 123 & 114 & 164 & 46 & 162 & 166 & 234 & 160 & 94 \\
\hline (2) & 39 & 58 & 227 & 18 & 14 & 205 & 82 & 184 & 132 & 204 & 110 & 72 & 228 & 141 & 172 & 111 \\
\hline (3) & 245 & 78 & 225 & 189 & 251 & 199 & 148 & 181 & 157 & 135 & 217 & 84 & 66 & 152 & 222 & 237 \\
\hline (4) & 92 & 106 & 129 & 255 & 196 & 125 & 89 & 59 & 213 & 219 & 226 & 51 & 29 & 90 & 36 & 11 \\
\hline (5) & 209 & 20 & 231 & 250 & 200 & 188 & 153 & 35 & 60 & 75 & 117 & 19 & 115 & 149 & 121 & 192 \\
\hline (6) & 26 & 43 & 145 & 7 & 102 & 85 & 4 & 235 & 8 & 240 & 221 & 173 & 215 & 64 & 220 & 91 \\
\hline (7) & 163 & 211 & 9 & 118 & 116 & 10 & 100 & 86 & 6 & 127 & 242 & 61 & 105 & 195 & 38 & 22 \\
\hline (8) & 97 & 185 & 244 & 0 & 98 & 203 & 218 & 137 & 63 & 155 & 126 & 76 & 23 & 107 & 161 & 93 \\
\hline (9) & 212 & 223 & 201 & 41 & 248 & 230 & 45 & 159 & 186 & 81 & 74 & 232 & 136 & 52 & 254 & 246 \\
\hline (10) & 2 & 239 & 104 & 108 & 144 & 247 & 56 & 24 & 120 & 44 & 83 & 176 & 194 & 170 & 179 & 69 \\
\hline (11) & 143 & 142 & 47 & 96 & 233 & 109 & 198 & 67 & 236 & 158 & 122 & 17 & 139 & 103 & 131 & 12 \\
\hline (12) & 174 & 70 & 15 & 33 & 147 & 124 & 112 & 42 & 50 & 30 & 34 & 16 & 214 & 210 & 241 & 37 \\
\hline (13) & 73 & 238 & 5 & 178 & 113 & 21 & 180 & 138 & 130 & 253 & 150 & 80 & 68 & 191 & 193 & 57 \\
\hline (14) & 140 & 182 & 48 & 154 & 28 & 169 & 177 & 49 & 31 & 25 & 65 & 187 & 119 & 134 & 167 & 168 \\
\hline (15) & 202 & 13 & 156 & 101 & 183 & 208 & 216 & 32 & 54 & 62 & 27 & 128 & 77 & 71 & 224 & 87 \\
\hline (16) & 40 & 171 & 95 & 3 & 206 & 53 & 207 & 151 & 99 & 175 & 133 & 79 & 146 & 55 & 190 & 252 \\
\hline
\end{tabular}

4.5. Differential Approximation Probability. The differential approximation probability $\mathrm{DP}_{f}$ can reflect the XOR distribution of the input and output of the Boolean function [6], that is, the maximum likelihood of outputting $\Delta y$, when the input is $\Delta x$,

$$
\begin{aligned}
& \mathrm{DP}_{f} \\
& \quad=\max _{\Delta x \neq 0, \Delta y}\left(\frac{\#\{x \in X \mid f(x) \oplus f(x \oplus \Delta x)=\Delta y\}}{2^{n}}\right),
\end{aligned}
$$

where $X$ denotes a set of all possible inputs and $2^{n}$ is the number of elements in the set.

The smaller the $\mathrm{DP}_{f}$, the stronger the ability of the S-box for fighting against differential cryptanalysis attacks, and vice versa.

4.6. Linear Approximation Probability. Given two randomly selected masks $\Gamma x$ and $\Gamma y$, we use $\Gamma x$ to calculate the mask of all possible values of an input $x$ and use $\Gamma y$ to calculate the mask of the output values $S(x)$ of the corresponding S-box. After masking the input and the output values, the maximum number of the same results is called the maximum linear approximation that can be computed by the following [46]:

$$
\mathrm{LP}=\max _{\Gamma x, \Gamma y \neq 0}\left|\frac{\#\{x \mid x \cdot \Gamma x=S(x) \cdot \Gamma y\}}{2^{n}}-\frac{1}{2}\right|,
$$

where $\Gamma x$ and $\Gamma y$ are the mask values of the input and output, respectively, and $S(x)$ is a set of all possible input values of $x$, the elements of which are $2^{n}$.

The smaller the LP, the stronger the ability of the S-box for fighting against linear cryptanalysis attacks, and vice versa.

\section{Cryptographic Criteria Analyses of Proposed S-Box}

To verify the generated S-box, we analyze its cryptographic criteria and compare it with many other S-boxes designed in [6-9, 25-41]. Moreover, our experimental environment is i3 CPU $5403.07 \mathrm{GHz}$, memory $2.00 \mathrm{GHz}$ Inter Core, Matlab2012b.

For initialization, we must choose the following parameters, $x_{0}=0.34, y_{0}=0.27, z_{0}=0.75, k_{1}=39.7, k_{2}=40.2$, $k_{3}=38.5, a=3.735, b=3.536, c=3.828, M_{0}=100, k=5$, $S=15, \mathrm{Ned}=3, \mathrm{Nre}=3, \mathrm{Nc}=8, \mathrm{Ns}=4$, Ped $=0.25$, $C=0.5, \alpha=0.2$. Calling the proposed algorithm with these parameters, we obtain the S-box that is depicted in Table 1.

5.1. Bijectivity Analysis. Use (8) to calculate the value of the objectivity of the S-box. Since the obtained bijectivity value of the proposed S-box is 128 that is the same as the desired value, we may conclude that our S-box satisfies the bijectivity.

5.2. Nonlinearity Analysis. Use (10) to calculate the nonlinearity of the S-box; Figure 3 depicts the statistical results of the nonlinearity of comparison algorithms. Table 6 shows the detailed nonlinearity results. From these results, we can see that the minimum nonlinearity value of eight Boolean functions of the proposed S-box is 106, and the average value 107.5 is greater than that of the other comparison algorithms. Thus, our S-box may have good nonlinearity and can resist the linear cryptanalysis.

5.3. Strict Avalanche Criterion Analysis. Table 2 depicts the dependence matrix of our S-box, and the SAC comparison results are shown in the third column of Table 6. From these two tables, we may conclude that our S-box satisfies the SAC. For our S-box, its obtained maximum SAC is 0.6094 , the minimum is 0.3750 , and its average value 0.5093 is close to the desired value 0.5 . Moreover, compared with the algorithm 


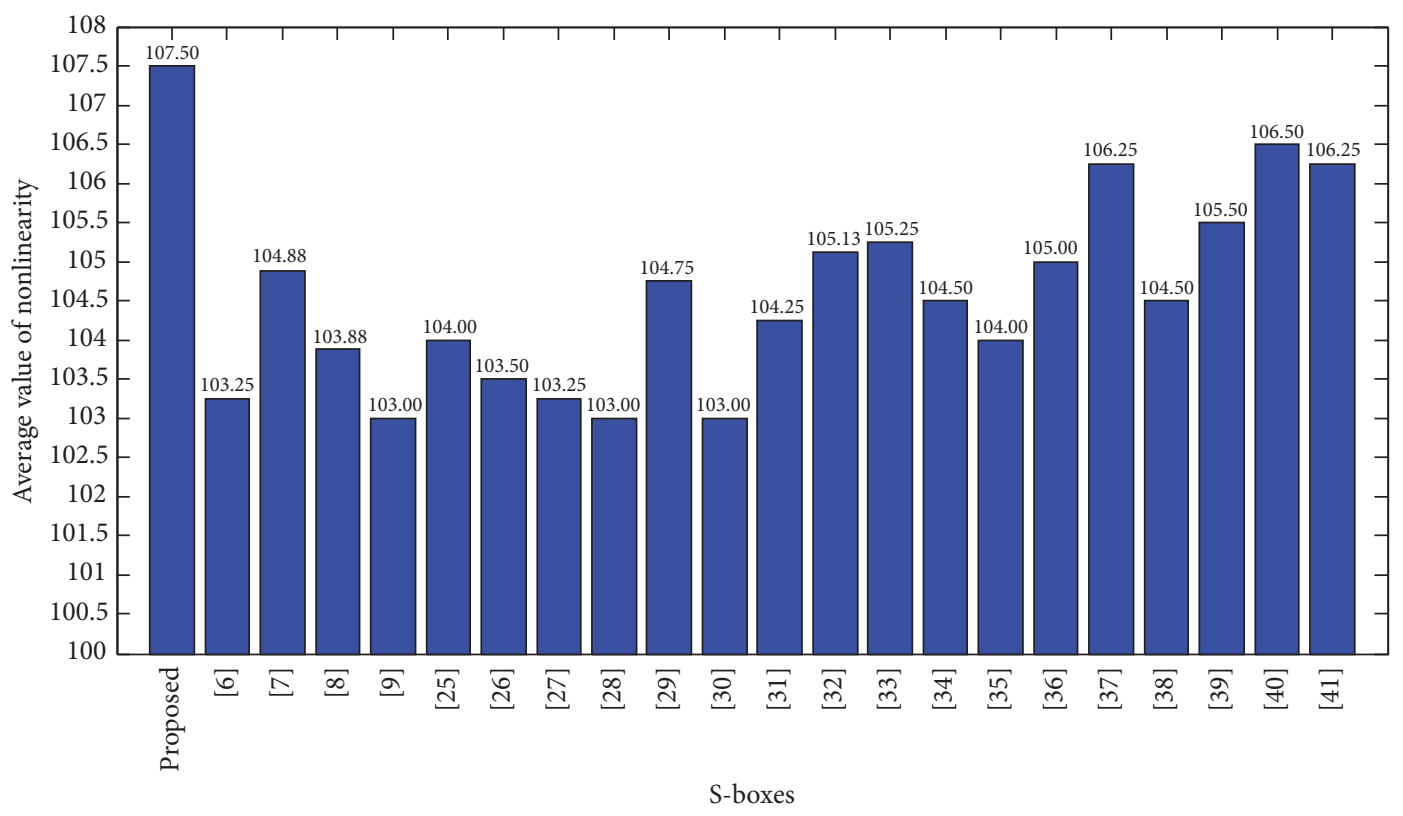

FIgURE 3: Statistical results of the nonlinearity.

TABLE 2: The dependence matrix of the generated S-box.

\begin{tabular}{lcccccccc}
\hline Number & $(1)$ & $(2)$ & $(3)$ & $(4)$ & $(5)$ & $(6)$ & $(7)$ & $(8)$ \\
\hline$(1)$ & 0.5313 & 0.4844 & 0.4844 & 0.5156 & 0.4844 & 0.5469 & 0.5313 & 0.5000 \\
$(2)$ & 0.4844 & 0.5469 & 0.5156 & 0.4688 & 0.4844 & 0.5156 & 0.5781 & 0.4844 \\
$(3)$ & 0.6094 & 0.4375 & 0.4844 & 0.5625 & 0.5625 & 0.4688 & 0.5000 & 0.5000 \\
$(4)$ & 0.4688 & 0.4844 & 0.5000 & 0.5469 & 0.5156 & 0.5156 & 0.5000 & 0.5625 \\
$(5)$ & 0.4219 & 0.5469 & 0.5000 & 0.5156 & 0.5000 & 0.5938 & 0.5313 & 0.5000 \\
$(6)$ & 0.4375 & 0.4688 & 0.5938 & 0.5000 & 0.5000 & 0.5156 & 0.5000 & 0.5000 \\
$(7)$ & 0.5000 & 0.4375 & 0.5000 & 0.5313 & 0.5781 & 0.5156 & 0.5156 & 0.5313 \\
$(8)$ & 0.5938 & 0.5000 & 0.3750 & 0.5313 & 0.4844 & 0.4844 & 0.5625 & 0.4531 \\
\hline
\end{tabular}

proposed in [40], the average value of the dependence matrix of the generated S-box is closer to the idea value 0.5.

5.4. Bit Independent Criterion Analysis. Tables 3 and 4 describe the BIC related criterion results of our S-box. The fourth and fifth columns of Table 6 depict the corresponding comparison results of the experimental results. Additionally, the average BIC-nonlinearity value of the proposed S-box is larger than that of S-box generation algorithms in $[7,8$, $28,31]$. The average BIC-SAC value of the proposed S-box is larger than that of algorithms in $[6-8,25,31-33,37-40]$. For our S-box, its average nonlinearity of the $f_{j} \oplus f_{k}$ is 103.07; the average correlation matrix is 0.5025 that is close to the desired value 0.5 . Therefore, we may obtain that our S-box has good bit independent criterion.

5.5. Differential Approximation Probability Analysis. Table 5 shows the differential probability result of our S-box calculated by (12), and the left of the right column in Table 6 depicts the DP comparison results of the experimental algorithms. Furthermore, Figure 4 depicts statistical results of the DP of comparison algorithms. These results show that the DP of the proposed S-box is not worse than that of the other comparison algorithms. It may indicate that our S-box can resist differential cryptanalysis because our S-box obtains a small differential probability, 0.0390 .

5.6. Linear Approximation Probability Analysis. In this subsection, we use (13) to calculate the linear probability of our S-box and compare it with other comparison algorithms. The linear probability results are shown in the right column of Table 6, from which we argue that our S-box may have a good ability to resist linear cryptanalysis. Besides, Figure 5 depicts the statistical results of the LP of comparison algorithms. These results show that the LP of the proposed S-box is not worse than that of algorithms in $[25,26,28,30,32,34,37,38$, 41].

Furthermore, Table 6 summarizes the above-mentioned evaluating criteria results of the experimental algorithms. From the table, we may make the following observations: (1) these chaotic based S-boxes have good cryptographic properties. (2) Comprehensively, our S-box, however, may have better encryption performance and can resist many modern cryptanalysis attacks to some extent. 
TABLE 3: BIC-nonlinearity criterion result of the generated S-box.

\begin{tabular}{lcccccccc}
\hline Number & $(1)$ & $(2)$ & $(3)$ & $(4)$ & $(5)$ & $(6)$ & $(7)$ & $(8)$ \\
\hline$(1)$ & 0 & 102 & 104 & 100 & 102 & 104 & 98 & 106 \\
$(2)$ & 102 & 0 & 102 & 102 & 100 & 102 & 106 & 106 \\
$(3)$ & 104 & 102 & 0 & 102 & 106 & 106 & 102 & 94 \\
$(4)$ & 100 & 102 & 102 & 0 & 100 & 104 \\
$(5)$ & 102 & 100 & 102 & 106 & 104 & 0 & 108 \\
$(6)$ & 104 & 102 & 106 & 102 & 108 & 100 & 100 \\
$(7)$ & 98 & 106 & 108 & 94 & 108 & 104 & 0 \\
$(8)$ & 106 & 106 & 100 & 104 & 104 & 104 \\
\hline
\end{tabular}

TABLE 4: BIC-SAC criterion result of the generate S-box.

\begin{tabular}{lcccccccc}
\hline Number & $(1)$ & $(2)$ & $(3)$ & $(4)$ & $(5)$ & $(6)$ & $(7)$ & $(8)$ \\
\hline$(1)$ & - & 0.5117 & 0.4961 & 0.5176 & 0.4941 & 0.4922 & 0.5000 & 0.4961 \\
$(2)$ & 0.5117 & - & 0.5117 & 0.4922 & 0.4941 & 0.4883 & 0.4863 & 0.5137 \\
$(3)$ & 0.4961 & 0.5117 & - & 0.5020 & 0.5000 & 0.5371 & 0.5000 & 0.5039 \\
$(4)$ & 0.5176 & 0.4922 & 0.5020 & - & 0.4922 & 0.4707 & 0.5156 & 0.4980 \\
$(5)$ & 0.4941 & 0.4941 & 0.5000 & 0.4922 & - & 0.5020 & 0.5156 \\
$(6)$ & 0.4922 & 0.4883 & 0.5371 & 0.4707 & 0.5020 & - & 0.5059 \\
$(7)$ & 0.5000 & 0.4863 & 0.5000 & 0.5156 & 0.5156 & 0.4941 & - & 0.5941 \\
$(8)$ & 0.4961 & 0.5137 & 0.5039 & 0.4980 & 0.5059 & 0.52540 & 0.5137 \\
\hline
\end{tabular}

TABLE 5: Differential approach table for the generated S-box.

\begin{tabular}{lccccccccccccccccc}
\hline Number & $(1)$ & $(2)$ & $(3)$ & $(4)$ & $(5)$ & $(6)$ & $(7)$ & $(8)$ & $(9)$ & $(10)$ & $(11)$ & $(12)$ & $(13)$ & $(14)$ & $(15)$ & $(16)$ \\
\hline$(1)$ & 6 & 6 & 6 & 6 & 6 & 8 & 6 & 8 & 8 & 8 & 6 & 6 & 6 & 6 & 6 & 6 \\
$(2)$ & 6 & 6 & 6 & 6 & 8 & 8 & 8 & 6 & 6 & 8 & 6 & 6 & 6 & 6 & 8 & 10 \\
$(3)$ & 6 & 8 & 6 & 6 & 8 & 6 & 6 & 6 & 6 & 6 & 6 & 6 & 8 & 8 & 6 & 8 \\
$(4)$ & 8 & 6 & 6 & 10 & 4 & 6 & 6 & 4 & 8 & 6 & 6 & 6 & 8 & 6 & 6 & 6 \\
$(5)$ & 6 & 8 & 6 & 6 & 6 & 8 & 6 & 6 & 10 & 6 & 6 & 6 & 6 & 6 & 6 & 8 \\
$(6)$ & 6 & 6 & 6 & 8 & 8 & 8 & 8 & 8 & 8 & 10 & 6 & 6 & 6 & 10 & 6 & 10 \\
$(7)$ & 8 & 6 & 8 & 8 & 8 & 6 & 6 & 6 & 8 & 8 & 6 & 6 & 4 & 6 & 8 & 8 \\
$(8)$ & 8 & 10 & 8 & 8 & 4 & 6 & 6 & 6 & 8 & 6 & 6 & 6 & 10 & 8 & 6 & 6 \\
$(9)$ & 6 & 6 & 6 & 6 & 8 & 8 & 8 & 8 & 6 & 8 & 6 & 6 & 6 & 8 & 8 & 8 \\
$(10)$ & 8 & 8 & 6 & 8 & 6 & 6 & 6 & 6 & 6 & 6 & 6 & 6 & 8 & 6 & 6 & 4 \\
$(11)$ & 6 & 6 & 8 & 6 & 6 & 6 & 8 & 4 & 6 & 8 & 6 & 6 & 6 & 6 & 6 & 8 \\
$(12)$ & 6 & 6 & 6 & 6 & 6 & 8 & 8 & 6 & 6 & 4 & 6 & 6 & 6 & 6 & 6 & 6 \\
$(13)$ & 8 & 8 & 6 & 8 & 6 & 8 & 8 & 6 & 6 & 8 & 10 & 6 & 6 & 8 & 6 & 6 \\
$(14)$ & 6 & 8 & 6 & 8 & 8 & 8 & 6 & 6 & 6 & 6 & 6 & 8 & 6 & 6 & 6 & 6 \\
$(15)$ & 8 & 6 & 6 & 6 & 6 & 6 & 6 & 6 & 6 & 6 & 8 & 6 & 6 & 6 & 6 \\
$(16)$ & 8 & 8 & 6 & 6 & 6 & 6 & 6 & 8 & 6 & 6 & 8 & 6 & 6 & 6 & 8 \\
\hline
\end{tabular}

\section{Conclusion}

This paper is concerned with developing a novel S-box generation algorithm, chaotic S-box based on the intertwining logistic map and bacterial foraging optimization. This algorithm firstly generates a set of intermediate S-boxes by iterating the chaotic map and then utilizes a bacterial foraging optimization algorithm to search for an S-box with good performance. During the search process, the nonlinearity and differential uniformity are involved to design the evaluation function. Experimental results investigate that the proposed S-box generation algorithm may generate an S-box with good cryptography characteristics. Since there are many other factors that affect the performance of S-boxes, for example, SAC, BIC, and LP, the investigation of applying them to design the evaluation function of the bacterial foraging optimization algorithm may be worth studying in the near future.

\section{List of Abbreviations}

S-box: Substitution box

BFO: Bacterial foraging optimization

SAC: Strict avalanche criterion 
TABLE 6: Cryptanalysis comparison results of S-boxes.

\begin{tabular}{|c|c|c|c|c|c|c|c|c|c|c|}
\hline \multirow{2}{*}{ S-boxes } & \multicolumn{3}{|c|}{ Nonlinearity } & \multicolumn{3}{|c|}{ SAC } & \multirow{2}{*}{ BIC-nonlinearity } & \multirow{2}{*}{ BIC-SAC } & \multirow{2}{*}{ DP } & \multirow{2}{*}{ LP } \\
\hline & Min & Max & Avg. & Min & Max & Avg. & & & & \\
\hline Proposed & 106 & 110 & 107.5 & 0.3750 & 0.6094 & 0.5093 & 103.07 & 0.5025 & 0.0390 & 0.1406 \\
\hline Ref. [6] & 100 & 108 & 103.250 & 0.3750 & 0.5938 & 0.5059 & 104.29 & 0.5031 & 0.0468 & 0.1250 \\
\hline Ref. [7] & 103 & 109 & 104.875 & 0.3984 & 0.5703 & 0.4966 & 102.96 & 0.5044 & 0.0390 & 0.1328 \\
\hline Ref. [8] & 101 & 108 & 103.875 & 0.3906 & 0.5781 & 0.5059 & 102.68 & 0.4958 & 0.0390 & 0.1328 \\
\hline Ref. [9] & 100 & 106 & 103 & 0.4219 & 0.6094 & 0.5000 & 103.14 & 0.5024 & 0.0546 & 0.1328 \\
\hline Ref. [25] & 102 & 106 & 104 & 0.3750 & 0.6094 & 0.4980 & 103.29 & 0.4971 & 0.0390 & 0.1484 \\
\hline Ref. [26] & 96 & 108 & 103.500 & 0.3906 & 0.5859 & 0.4939 & 103.64 & 0.4992 & 0.0390 & 0.1523 \\
\hline Ref. [27] & 100 & 106 & 103.250 & 0.4219 & 0.5938 & 0.5049 & 103.71 & 0.5010 & 0.0390 & 0.1328 \\
\hline Ref. [28] & 96 & 106 & 103 & 0.3906 & 0.6250 & 0.5039 & 100.36 & 0.5010 & 0.0390 & 0.1484 \\
\hline Ref. [29] & 102 & 108 & 104.750 & 0.3906 & 0.5938 & 0.5056 & 104.07 & 0.5022 & 0.0468 & 0.1250 \\
\hline Ref. [30] & 98 & 108 & 103 & 0.4063 & 0.5938 & 0.5012 & 104.07 & 0.4989 & 0.0468 & 0.1484 \\
\hline Ref. [31] & 98 & 108 & 104.25 & 0.2813 & 0.6094 & 0.4954 & 102.86 & 0.5048 & 0.0468 & 0.1406 \\
\hline Ref. [32] & 103 & 109 & 105.125 & 0.4141 & 0.6094 & 0.5061 & 103.68 & 0.4983 & 0.0390 & 0.1562 \\
\hline Ref. [33] & 102 & 108 & 105.250 & 0.4063 & 0.5781 & 0.5059 & 104.29 & 0.5029 & 0.0468 & 0.1250 \\
\hline Ref. [34] & 100 & 108 & 104.5 & 0.4219 & 0.6094 & 0.4978 & 103.64 & 0.5010 & 0.0468 & 0.1406 \\
\hline Ref. [35] & 100 & 106 & 104 & 0.3750 & 0.6250 & 0.4946 & 103.21 & 0.5019 & 0.0390 & 0.1328 \\
\hline Ref. [36] & 98 & 108 & 105 & 0.4219 & 0.5781 & 0.4917 & 106.07 & 0.4997 & 0.1250 & 0.1171 \\
\hline Ref. [37] & 104 & 110 & 106.25 & 0.4219 & 0.5938 & 0.5039 & 103.36 & 0.5059 & 0.0390 & 0.1406 \\
\hline Ref. [38] & 101 & 107 & 104.5 & 0.4219 & 0.5781 & 0.4963 & 103.29 & 0.4938 & 0.0390 & 0.1406 \\
\hline Ref. [39] & 100 & 110 & 105.50 & 0.4063 & 0.6094 & 0.5010 & 103.79 & 0.5036 & 0.0468 & 0.1328 \\
\hline Ref. [40] & 104 & 110 & 106.5 & 0.4375 & 0.6406 & 0.5120 & 104.57 & 0.5042 & 0.0390 & 0.1328 \\
\hline Ref. [41] & 104 & 108 & 106.25 & 0.3906 & 0.5625 & 0.4949 & 103.64 & 0.5007 & 0.0390 & 0.1406 \\
\hline
\end{tabular}

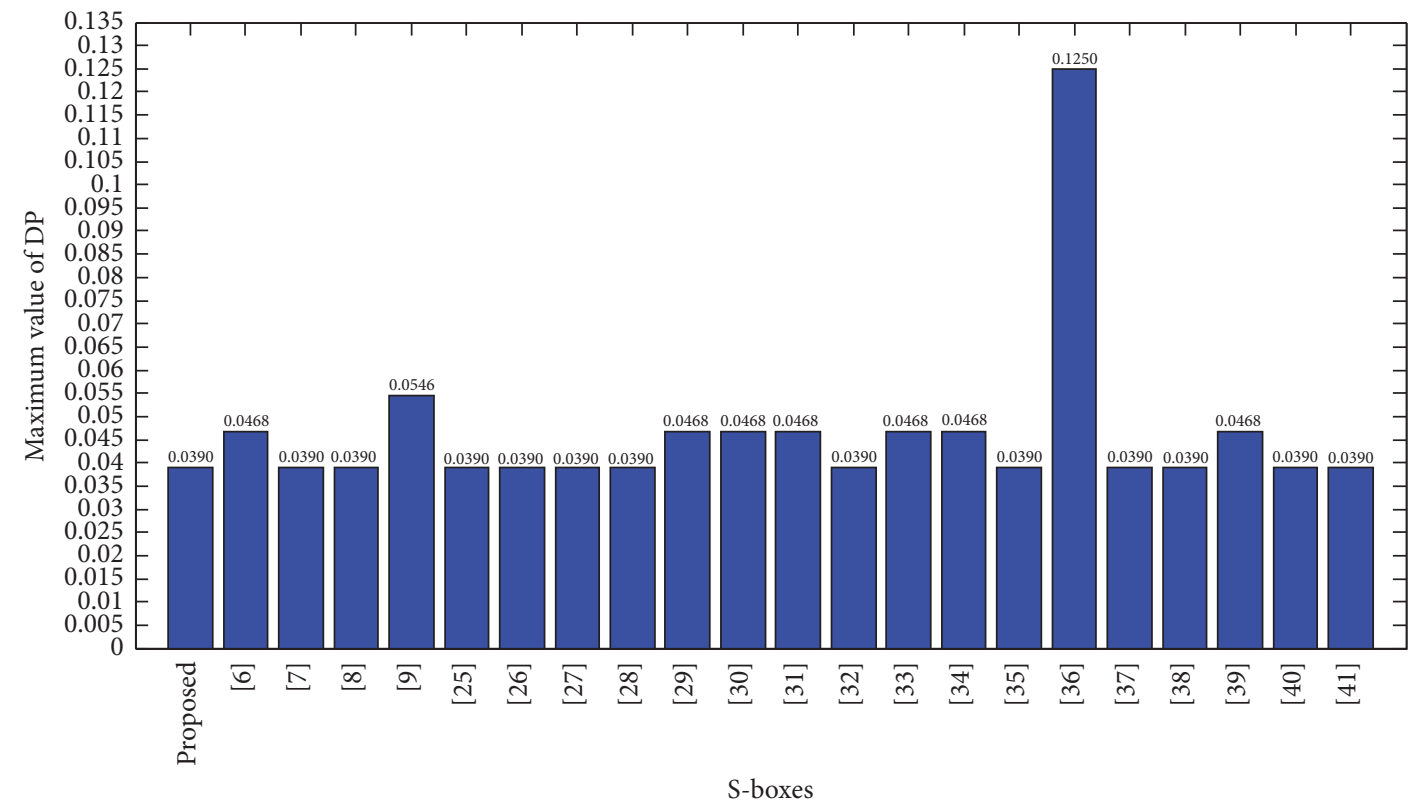

FIgURE 4: Statistical results of the DP.

BIC: Bit independent criterion

DP: Differential approximation probability

LP: Linear approximation probability.

\section{Conflicts of Interest}

The authors declare no conflicts of interest. 


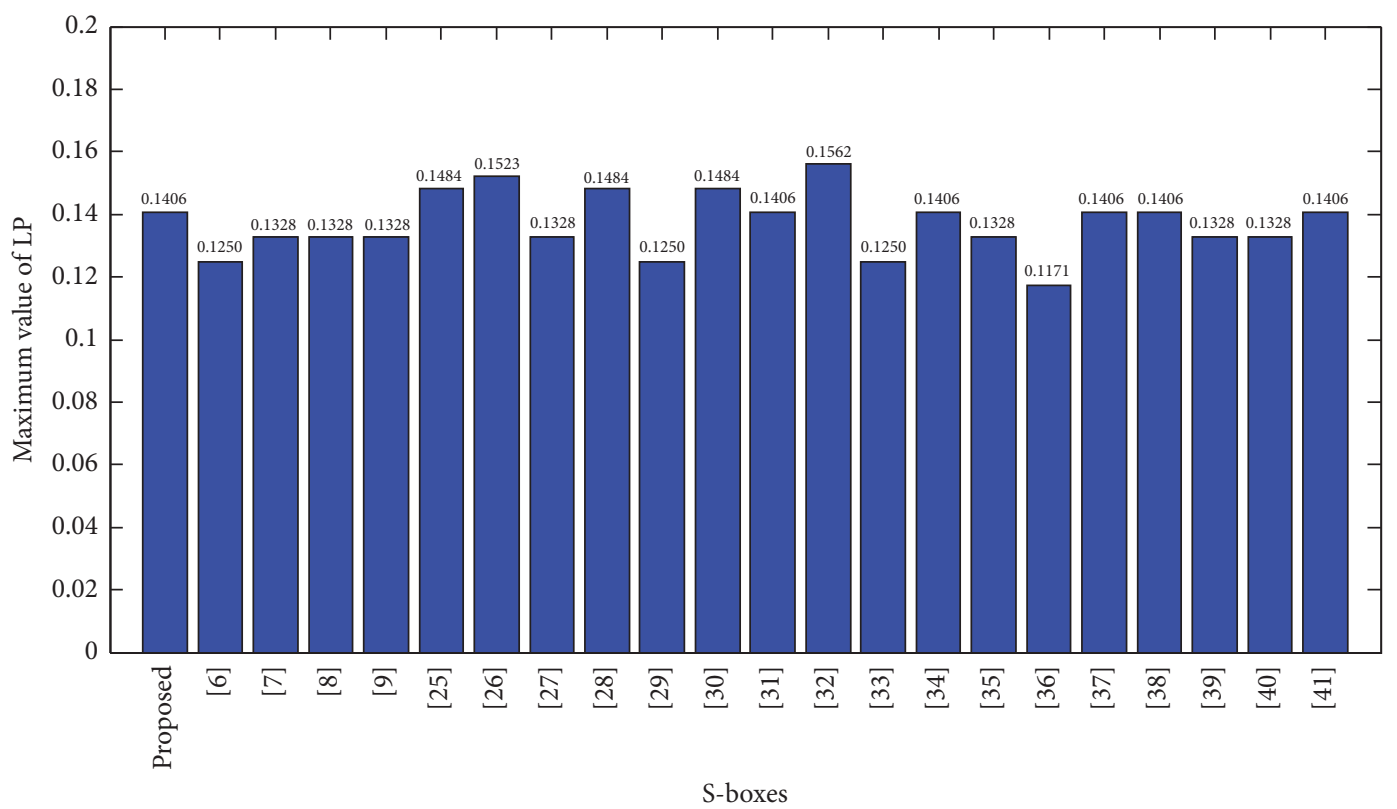

FIGURE 5: Statistical results of the LP.

\section{Acknowledgments}

The authors would like to acknowledge the financial supports by the National Natural Science Foundation of China (nos. 60603092, 60975042, and 51472066).

\section{References}

[1] X. Wang and Q. Wang, "A novel image encryption algorithm based on dynamic S-boxes constructed by chaos," Nonlinear Dynamics, vol. 75, no. 3, pp. 567-576, 2014.

[2] I. Hussain, T. Shah, and M. A. Gondal, "Application of Sbox and chaotic map for image encryption," Mathematical and Computer Modelling, vol. 57, no. 9-10, pp. 2576-2579, 2013.

[3] X. Zhang, Y. Mao, and Z. Zhao, "An efficient chaotic image encryption based on alternate circular S-boxes," Nonlinear Dynamics, vol. 78, no. 1, pp. 359-369, 2014.

[4] H. Liu, A. Kadir, and Y. Niu, "Chaos-based color image block encryption scheme using S-box," AEÜ - International Journal of Electronics and Communications, vol. 68, no. 7, pp. 676-686, 2014.

[5] I. Hussain and M. A. Gondal, "An extended image encryption using chaotic coupled map and S-box transformation," Nonlinear Dynamics, vol. 76, no. 2, pp. 1355-1363, 2014.

[6] G. Jakimoski and L. c. Kocarev, "Chaos and cryptography: block encryption ciphers based on chaotic maps," IEEE Transactions on Circuits and Systems I: Fundamental Theory and Applications, vol. 48, no. 2, pp. 163-169, 2001.

[7] G. Tang, X. Liao, and Y. Chen, "A novel method for designing S-boxes based on chaotic maps," Chaos, Solitons \& Fractals, vol. 23, no. 2, pp. 413-419, 2005.

[8] G. Tang and X. Liao, "A method for designing dynamical Sboxes based on discretized chaotic map," Chaos, Solitons \& Fractals, vol. 23, no. 5, pp. 1901-1909, 2005.

[9] G. Chen, Y. Chen, and X. Liao, "An extended method for obtaining S-boxes based on three-dimensional chaotic baker maps," Chaos, Solitons \& Fractals, vol. 31, no. 3, pp. 571-579, 2007.

[10] S. Behnia, A. Akhshani, H. Mahmodi, and A. Akhavan, "A novel algorithm for image encryption based on mixture of chaotic maps," Chaos, Solitons \& Fractals, vol. 35, no. 2, pp. 408-419, 2008.

[11] L. Nian-sheng, "Pseudo-randomness and complexity of binary sequences generated by the chaotic system," Communications in Nonlinear Science and Numerical Simulation, vol. 16, no. 2, pp. 761-768, 2011.

[12] X.-Y. Wang and X. Qin, "A new pseudo-random number generator based on CML and chaotic iteration," Nonlinear Dynamics, vol. 70, no. 2, pp. 1589-1592, 2012.

[13] M. Francois, T. Grosges, D. Barchiesi, and R. Erra, "Pseudorandom number generator based on mixing of three chaotic maps," Communications in Nonlinear Science and Numerical Simulation, vol. 19, no. 4, pp. 887-895, 2014.

[14] J. Khan, J. Ahmad, and S. O. Hwang, "An efficient image encryption scheme based on: Henon map, skew tent map and S-Box," in Proceedings of the 6th International Conference on Modeling, Simulation, and Applied Optimization, ICMSAO 2015, pp. 1-6, May 2015.

[15] M. Ahmad, H. Haleem, and P. M. Khan, "A new chaotic substitution box design for block ciphers," in Proceedings of the 1st International Conference on Signal Processing and Integrated Networks, SPIN 2014, pp. 255-258, February 2014.

[16] J. Peng, S. Jin, L. Lei, and X. Liao, "Construction and analysis of dynamic S-boxes based on spatiotemporal chaos," in Proceedings of the IEEE 11th International Conference on Cognitive Informatics \& Cognitive Computing (ICCI* CC), pp. 274-278, 2012.

[17] Y. Wang, K.-W. Wong, C. Li, and Y. Li, "A novel method to design S-box based on chaotic map and genetic algorithm," Physics Letters A, vol. 376, no. 6-7, pp. 827-833, 2012.

[18] S. M. Abd-Elazim and E. S. Ali, "Power system stability enhancement via bacteria foraging optimization algorithm," 
Arabian Journal for Science and Engineering, vol. 38, no. 3, pp. 599-611, 2013.

[19] E. S. Ali and S. M. Abd-Elazim, "Bacteria foraging optimization algorithm based load frequency controller for interconnected power system," International Journal of Electrical Power \& Energy Systems, vol. 33, no. 3, pp. 633-638, 2011.

[20] S. M. Abd-Elazim and E. S. Ali, "Synergy of particle swarm optimization and bacterial foraging for TCSC damping controller design," WSEAS Transactions on Power Systems, vol. 8, no. 2, pp. 74-84, 2013.

[21] C. Sur and A. Shukla, "Discrete bacteria foraging optimization algorithm for vehicle distribution optimization in graph based road network management," Advances in Intelligent Systems and Computing, vol. 235, pp. 351-358, 2014.

[22] M. Tripathy and S. Mishra, "Bacteria foraging-based solution to optimize both real power loss and voltage stability limit," IEEE Transactions on Power Systems, vol. 22, no. 1, pp. 240-248, 2007.

[23] I. S. Sam, P. Devaraj, and R. S. Bhuvaneswaran, "An intertwining chaotic maps based image encryption scheme," Nonlinear Dynamics, vol. 69, no. 4, pp. 1995-2007, 2012.

[24] K. M. Passino, "Biomimicry of bacterial foraging for distributed optimization and control," IEEE Control Systems Magazine, vol. 22, no. 3, pp. 52-67, 2002.

[25] G. Chen, "A novel heuristic method for obtaining S-boxes," Chaos, Solitons \& Fractals, vol. 36, no. 4, pp. 1028-1036, 2008.

[26] M. Asim and V. Jeoti, "Efficient and simple method for designing chaotic S-boxes," ETRI Journal, vol. 30, no. 1, pp. 170-172, 2008.

[27] F. Özkaynak and A. B. Özer, "A method for designing strong SBoxes based on chaotic Lorenz system," Physics Letters A, vol. 374, no. 36, pp. 3733-3738, 2010.

[28] M. Khan, T. Shah, H. Mahmood, M. A. Gondal, and I. Hussain, "A novel technique for the construction of strong S-boxes based on chaotic Lorenz systems," Nonlinear Dynamics, vol. 70, no. 3, pp. 2303-2311, 2012.

[29] I. Hussain, T. Shah, and M. A. Gondal, "A novel approach for designing substitution-boxes based on nonlinear chaotic algorithm," Nonlinear Dynamics, vol. 70, no. 3, pp. 1791-1794, 2012.

[30] M. Khan, T. Shah, H. Mahmood, and M. A. Gondal, "An efficient method for the construction of block cipher with multichaotic systems," Nonlinear Dynamics, vol. 71, no. 3, pp. 489492, 2013.

[31] M. Khan, T. Shah, and M. A. Gondal, "An efficient technique for the construction of substitution box with chaotic partial differential equation," Nonlinear Dynamics, vol. 73, no. 3, pp. 1795-1801, 2013.

[32] F. Özkaynak and S. Yavuz, "Designing chaotic S-boxes based on time-delay chaotic system," Nonlinear Dynamics, vol. 74, no. 3 , pp. 551-557, 2013.

[33] I. Hussain, T. Shah, M. A. Gondal, and H. Mahmood, "A novel method for designing nonlinear component for block cipher based on TD-ERCS chaotic sequence," Nonlinear Dynamics, vol. 73, no. 1-2, pp. 633-637, 2013.

[34] M. Khan and T. Shah, "An efficient construction of substitution box with fractional chaotic system," Signal, Image and Video Processing, vol. 9, no. 6, pp. 1335-1338, 2015.

[35] M. Khan and T. Shah, "A construction of novel chaos base nonlinear component of block cipher," Nonlinear Dynamics, vol. 76, no. 1, pp. 377-382, 2014.
[36] M. Khan and T. Shah, "A novel construction of substitution box with Zaslavskii chaotic map and symmetric group," Journal of Intelligent \& Fuzzy Systems: Applications in Engineering and Technology, vol. 28, no. 4, pp. 1509-1517, 2015.

[37] Ü. Çavuşoğlu, A. Zengin, I. Pehlivan, and S. Kaçar, "A novel approach for strong S-Box generation algorithm design based on chaotic scaled Zhongtang system," Nonlinear Dynamics, vol. 87, no. 2, pp. 1081-1094, 2017.

[38] F. Özkaynak, V. Çelik, and A. B. Özer, "A new S-box construction method based on the fractional-order chaotic Chen system," Signal, Image and Video Processing, vol. 11, no. 4, pp. 659-664, 2017.

[39] A. Belazi and A. A. A. El-Latif, "A simple yet efficient Sbox method based on chaotic sine map," Optik - International Journal for Light and Electron Optics, vol. 130, pp. 1438-1444, 2017.

[40] T. Farah, R. Rhouma, and S. Belghith, "A novel method for designing S-box based on chaotic map and Teaching-LearningBased Optimization," Nonlinear Dynamics, vol. 88, no. 2, pp. 1059-1074, 2017.

[41] Y. Tian and Z. Lu, "S-box: L-L cascade chaotic map and line map," in Proceedings of the International Conference on Image and Graphics, pp. 297-309, Springer, Cham, Switzerland, 2015.

[42] X. J. Bi, L. Sheng, and J. Chen, "S-box optimization design based on improved particle swarm optimization algorithm," Computer Engineering, vol. 37, no. 23, pp. 149-151, 2011 (Chinese).

[43] B. X. Lu, Research on Optimization and Improvement of S-box in Block Cipher, Southwest Jiaotong University, Sichuan, China, 2013.

[44] C. Adams and S. Tavares, "Good S-boxes are easy to find," in Advances in Cryptology-CRYPTO'89 Proceedings, pp. 612-615, Springer, New York, NY, USA, 1990.

[45] A. F. Webster and S. E. Tavares, "On the design of S-boxes," in Advances in Cryptology-CRYPTO'85 Proceedings, pp. 523-534, Springer, Berlin, Germany, 1986.

[46] M. Matsui, "Linear cryptanalysis method of DES cipher," in Advances in Cryptology, Proc. Eurocrypt'93, Proceedings, pp. 386-397, Springer, Berlin, Germany, 1994. 


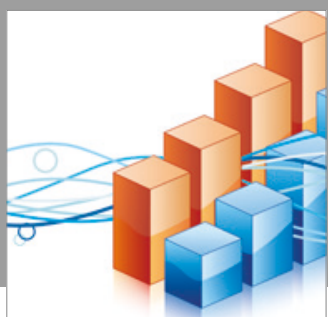

Advances in

Operations Research

vatersals

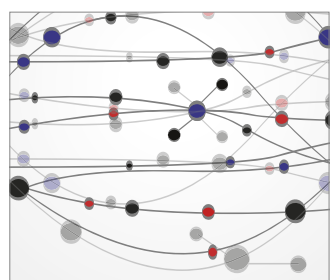

\section{The Scientific} World Journal
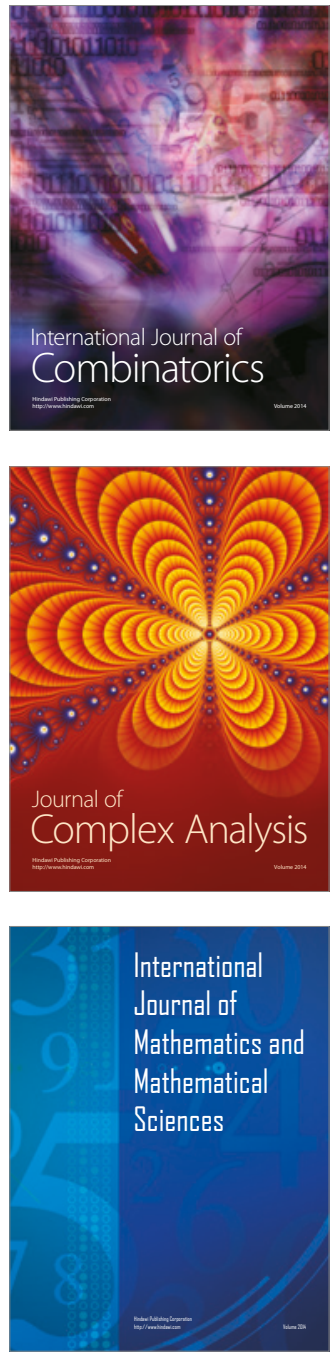
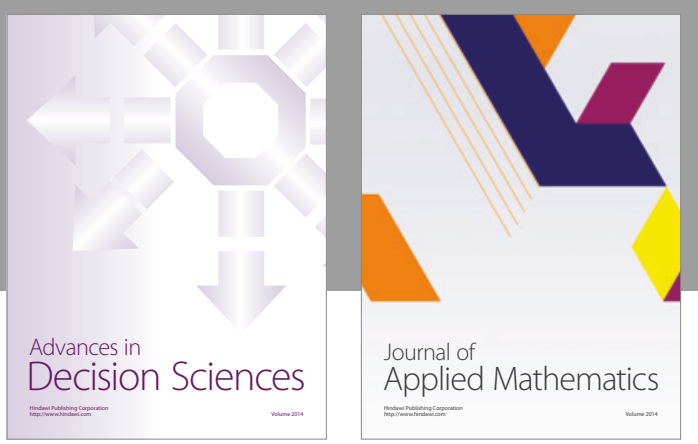

Algebra

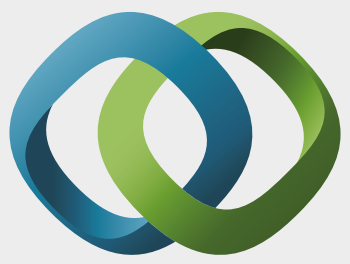

\section{Hindawi}

Submit your manuscripts at

https://www.hindawi.com
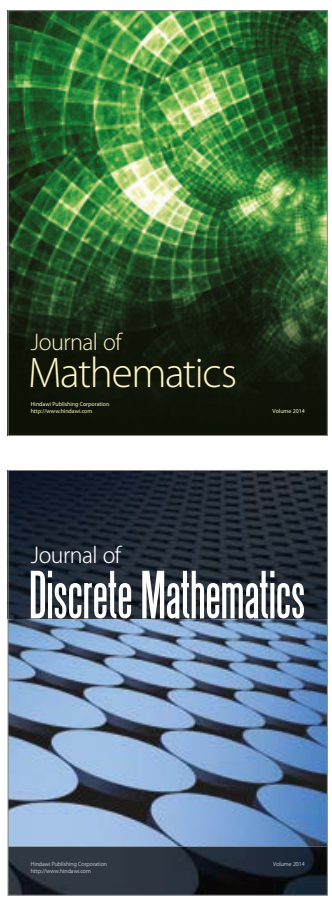

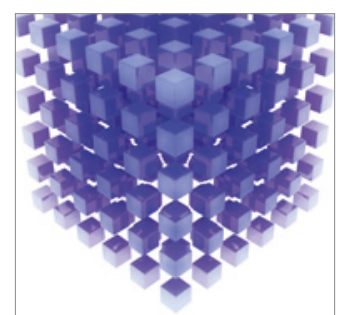

Mathematical Problems in Engineering
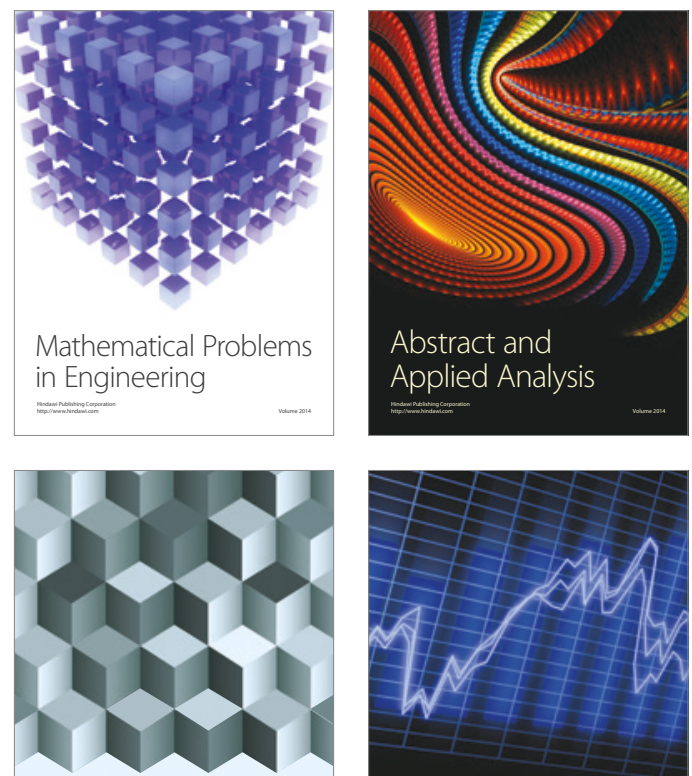

Journal of

Function Spaces

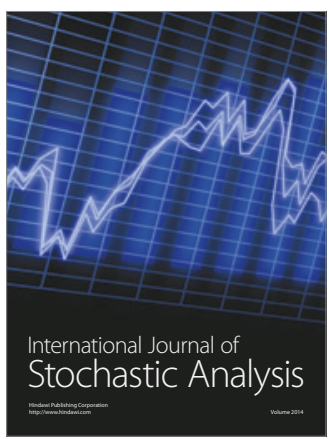

Probability and Statistics
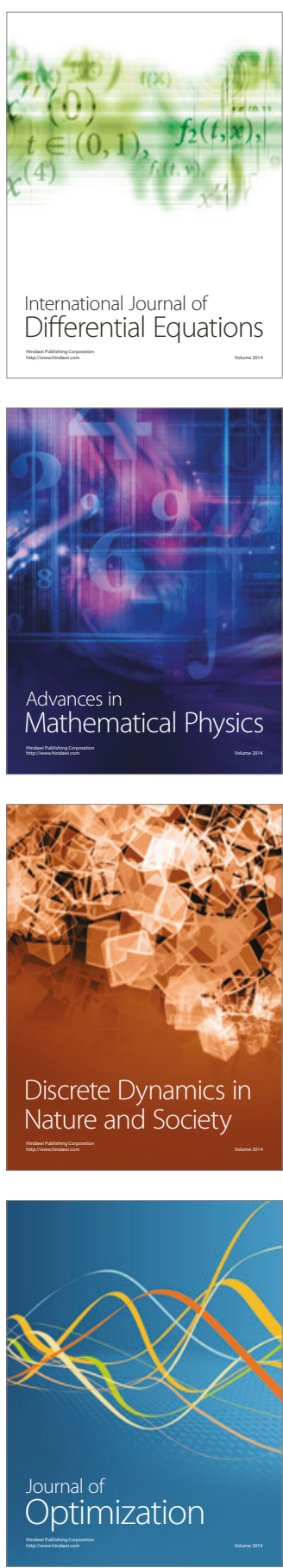